\title{
Azimuthal Asymmetries of the Drell-Yan Process in pA Collisions
}

\author{
Jian-Hua Gao \\ Shandong Provincial Key Laboratory of Optical Astronomy and Solar-Terrestrial Environment, \\ Institute of Space Sciences, Shandong University, Weihai 264209, China \\ gaojh@sdu.edu.cn \\ Key Laboratory of Quark and Lepton Physics (MOE), Central China Normal University, \\ Wuhan 430079, China
}

Published 29 February 2016

\begin{abstract}
We discuss the azimuthal asymmetries of the Drell-Yan process in nucleon-nucleus collisions at the low transverse momentum of the lepton pair. Within the transversemomentum-dependent (TMD) factorization formalism, the nuclear effects of these azimuthal asymmetries can be from the gauge link of the TMD quark distribution. We estimate all these nuclear effects within the assumption that all the TMD parton distributions or correlations are in Gaussian forms.
\end{abstract}

\section{Introduction}

It is well known that multiple scattering of a propagating parton inside a medium can result in many interesting phenomena such as transverse momentum broadening and parton energy loss. ${ }^{1-6}$ However, the multiple parton interaction could also lead to another interesting phenomena relevant to azimuthal asymmetries of final states when nuclei are involved in high energy scattering. This provides another possible good probe to detect the properties of the hot or cold nuclear matter. The nuclear dependence of the azimuthal asymmetry in semi-inclusive deep inelastic scattering and Drell-Yan process has been studied recently. ${ }^{7-10}$ In this report, we will give a brief and compact discussion on the azimuthal asymmetries of the Drell-Yan process in nucleon-nucleus collisions at the low transverse momentum of the lepton pair.

\section{Azimuthal Asymmetries in Drell-Yan Process}

In the kinematic region where the transverse momentum of the lepton pair is much lower than the mass $Q$ of the lepton pair, the differential cross section can be

This is an Open Access article published by World Scientific Publishing Company. It is distributed under the terms of the Creative Commons Attribution 3.0 (CC-BY) License. Further distribution of this work is permitted, provided the original work is properly cited. 
calculated in the framework of the TMD factorization theorem. ${ }^{11,12}$ The results for hadron-hadron collisions have been given in Refs.. ${ }^{13-15}$ We will work in the Collins-Soper frame in which the cross section for the single polarized nucleon after integrating over polar angle can be expressed as

$$
\begin{aligned}
& \frac{d \sigma}{d \phi d^{2} q_{T} d x_{1} d x_{2}}=\frac{\alpha_{e m}^{2}}{3 Q^{2}}\left\{2 \mathcal{F}_{0}\left[f_{1}, f_{1}\right]+\cos 2 \phi \mathcal{F}_{1}\left[h_{1}^{\perp}, h_{1}^{\perp}\right]\right. \\
& \quad+\lambda_{s} \sin 2 \phi \mathcal{F}_{1}\left[h_{1 L}^{\perp}, h_{1}^{\perp}\right]-2\left|\vec{s}_{T}\right| \sin \phi_{s} \mathcal{F}_{2}\left[f_{1 T}^{\perp}, f_{1}\right] \\
& \left.+\left|\vec{s}_{T}\right| \sin \left(2 \phi-\phi_{s}\right) \mathcal{F}_{3}\left[h_{1}, h_{1}^{\perp}\right]+\left|\vec{s}_{T}\right| \sin \left(2 \phi+\phi_{s}\right) \mathcal{F}_{4}\left[h_{1 T}^{\perp}, h_{1}^{\perp}\right]\right\} .
\end{aligned}
$$

where $\lambda_{s}$ and $\vec{s}_{T}$ are, respectively, the helicity and the transverse polarization vector of the polarizaed nucleon; $\phi$ and $\phi_{s}$ are, respectively, azimuthal angles of the lepton pair and azimuthal angle of the polarization vector of the nucleon with respect to the hadron plane. We see that the differential cross section is determined by six TMD quark and anti-quark distributions and correlation functions $f_{1}\left(x, \vec{k}_{T}\right), f_{1 T}^{\perp}\left(x, \vec{k}_{T}\right)$, $h_{1}^{\perp}\left(x, \vec{k}_{T}\right), h_{1}\left(x, \vec{k}_{T}\right), h_{1 T}^{\perp}\left(x, \vec{k}_{T}\right)$, and $h_{1 L}^{\perp}\left(x, \vec{k}_{T}\right)$. The $\mathcal{F}_{j}$ 's $(j=0$ through 4) are functionals of quark distributions or correlation functions which we refer the reader to in Ref. ${ }^{10}$ for details. There are five different azimuthal asymmetries $\cos 2 \phi, \sin 2 \phi$, $\sin \phi_{s}, \sin \left(2 \phi-\phi_{s}\right)$, and $\sin \left(2 \phi+\phi_{s}\right)$, which can be defined as, respectively,

$$
\begin{aligned}
A^{\cos 2 \phi} & =\frac{\mathcal{F}_{1}\left[h_{1}^{\perp}, h_{1}^{\perp}\right]}{4 \mathcal{F}_{0}\left[f_{1}, f_{1}\right]}, A^{\sin 2 \phi}=\lambda_{s} \frac{\mathcal{F}_{1}\left[h_{1 L}^{\perp}, h_{1}^{\perp}\right]}{4 \mathcal{F}_{0}\left[f_{1}, f_{1}\right]}, A^{\sin \phi_{s}}=-\left|\vec{s}_{T}\right| \frac{\mathcal{F}_{2}\left[f_{1 T}^{\perp}, f_{1}\right]}{2 \mathcal{F}_{0}\left[f_{1}, f_{1}\right]} \\
A^{\sin \left(2 \phi-\phi_{s}\right)} & =\left|\vec{s}_{T}\right| \frac{\mathcal{F}_{3}\left[h_{1}, h_{1}^{\perp}\right]}{4 \mathcal{F}_{0}\left[f_{1}, f_{1}\right]}, A^{\sin \left(2 \phi+\phi_{s}\right)}=\left|\vec{s}_{T}\right| \frac{\mathcal{F}_{4}\left[h_{1 T}^{\perp}, h_{1}^{\perp}\right]}{4 \mathcal{F}_{0}\left[f_{1}, f_{1}\right]},
\end{aligned}
$$

Therefore the nuclear effects of azimuthal asymmetries can be measured by the ratio of nucleon-nucleus collisions (NA) to nucleon-nucleon ones (NN):

$$
\begin{aligned}
R^{\cos 2 \phi} & =\frac{A_{N A}^{\cos 2 \phi}}{A_{N N}^{\cos 2 \phi}}, \quad R^{\sin 2 \phi}=\frac{A_{N A}^{\sin 2 \phi}}{A_{N N}^{\sin 2 \phi}}, \quad R^{\sin \phi_{s}}=\frac{A_{N A}^{\sin \phi_{s}}}{A_{N N}^{\sin \phi_{s}}}, \\
R^{\sin \left(2 \phi-\phi_{s}\right)} & =\frac{A_{N A}^{\sin \left(2 \phi-\phi_{s}\right)}}{A_{N N}^{\sin \left(2 \phi-\phi_{s}\right)}}, R^{\sin \left(2 \phi+\phi_{s}\right)}=\frac{A_{N A}^{\sin \left(2 \phi+\phi_{s}\right)}}{A_{N N}^{\sin \left(2 \phi+\phi_{s}\right)}} .
\end{aligned}
$$

\section{Nuclear Effects of the Azimuthal Asymmetries}

It has been shown in Ref. ${ }^{7}, 16$ that the gauge link defined in the TMD quark distribution or correlation functions can lead to a strong nuclear dependence. How these nuclear effects appear in Drell-Yan process is illustrated in Fig. 1. The gauge link is produced from the initial interaction between a quark or antiquark from one nucleon and the other nucleon or nucleus. The difference of the nucleon-nucleon interaction (left panel in Fig. 1) and nucleon-nucleus interaction (right panel in Fig. 1) is that the gluons interchanged between the quark and the nucleus can be attached to different nucleons in the nucleus. Such difference is just where the nuclear effect would come from. Due to the color confinement, we require at least two gluons to 


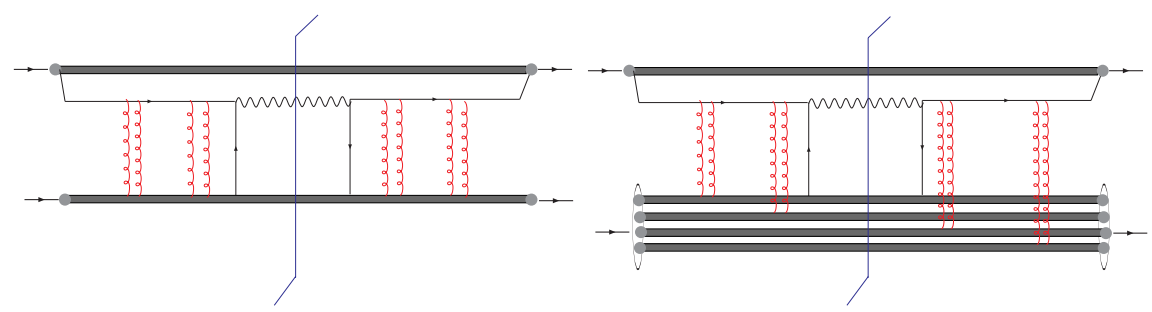

Fig. 1. (Color online) The difference of the gauge links in Drell-Yan process between the nucleonnucleon collisions and nucleon-nucleus collisions

be attached to one nucleon to make color singlet. Besides, we require different pairs of gluons to different nucleons as many as possible in order to obtain the maximal nuclear effects. Together with the approximation that the nucleus is very large and weak interacting, we can relate the quark distribution matrix in a nucleus to the one in the nucleon

$$
\Phi^{A}\left(x, \vec{k}_{T}\right) \approx \frac{A}{\pi \Delta_{2 F}} \int d^{2} l_{T} e^{-\left(\vec{k}_{T}-\vec{l}_{T}\right)^{2} / \Delta_{2 F}} \Phi^{N}\left(x, \vec{l}_{T}\right)
$$

where the only parameter $\Delta_{2 F}$ denotes the total average squared transverse momentum broadening and the components of $\Phi\left(x, \vec{k}_{T}\right)$ are defined by,

$$
\Phi_{\alpha \beta}\left(x, \vec{k}_{T}\right) \equiv \int \frac{p^{+} d y^{-}}{2 \pi} \frac{d^{2} y_{\perp}}{(2 \pi)^{2}} e^{i x p^{+} y^{-}-i \vec{k}_{\perp} \cdot \vec{y}_{\perp}}\left\langle N\left|\bar{\psi}_{\beta}(0) \mathcal{L}(0, y) \psi_{\alpha}(y)\right| N\right\rangle,
$$

where $\mathcal{L}(0, y)$ is the gauge link we mentioned above which is necessary to ensure the gauge invariance of the matrix. If we take the Gaussian ansatz for the transverse momentum dependence, i.e.,

$$
\begin{gathered}
f_{1}^{N}\left(x, \vec{k}_{T}\right)=\frac{1}{\pi \alpha} f_{1}^{N}(x) e^{-\frac{\vec{k}_{T}^{2}}{\alpha}}, \quad h_{1}^{N \perp}\left(x, \vec{k}_{T}\right)=\frac{1}{\pi \beta} h_{1}^{N \perp}(x) e^{-\frac{\vec{k}_{T}^{2}}{\beta}}, \\
h_{1 L}^{N \perp}\left(x, \vec{k}_{T}\right)=\frac{1}{\pi \sigma_{1}} h_{1 L}^{N \perp}(x) e^{\frac{-\vec{k}_{T}^{2}}{\sigma_{1}}}, \quad f_{1 T}^{N \perp}\left(x, \vec{k}_{T}\right)=\frac{1}{\pi \sigma_{2}} f_{1 T}^{N \perp}(x) e^{\frac{-\vec{k}_{T}^{2}}{\sigma_{2}}}, \\
h_{1}^{N}\left(x, \vec{k}_{T}\right)=\frac{1}{\pi \sigma_{3}} h_{1}^{N}(x) e^{\frac{-\vec{k}_{T}^{2}}{\sigma_{3}}}, \quad h_{1 T}^{N \perp}\left(x, \vec{k}_{T}\right)=\frac{1}{\pi \sigma_{4}} h_{1 T}^{N \perp}(x) e^{\frac{-\vec{k}_{T}^{2}}{\sigma_{4}}} .
\end{gathered}
$$

where we have assumed different flavors have the same Gaussian widths for the same types of TMD distributions and we have suppressed the flavor index. If we assume the Gaussian widths of different TMD distributions differ very little, which means

$$
\begin{aligned}
& \delta_{0} \equiv \beta-\alpha \ll \alpha, \quad \delta_{1} \equiv \sigma_{1}-\alpha \ll \alpha, \quad \delta_{2} \equiv \sigma_{2}-\alpha \ll \alpha, \\
& \delta_{3} \equiv \sigma_{3}-\alpha \ll \alpha, \quad \delta_{4} \equiv \sigma_{4}-\alpha \ll \alpha,
\end{aligned}
$$

we can have

$$
R^{\cos 2 \phi} \approx\left(\frac{2 \alpha}{2 \alpha+\Delta_{2 F}}\right)^{2}\left[1+\frac{\Delta_{2 F}}{2 \alpha+\Delta_{2 F}}\left(\frac{3}{2}-\frac{q_{T}^{2}}{2 \alpha+\Delta_{2 F}}-\frac{q_{T}^{2}}{2 \alpha}\right) \frac{\delta_{0}}{\alpha}\right],
$$




$$
\begin{aligned}
& R^{\sin 2 \phi} \approx\left(\frac{2 \alpha}{2 \alpha+\Delta_{2 F}}\right)^{2}\left[1+\frac{\Delta_{2 F}}{2 \alpha+\Delta_{2 F}}\left(\frac{3}{2}-\frac{q_{T}^{2}}{2\left(2 \alpha+\Delta_{2 F}\right)}-\frac{q_{T}^{2}}{4 \alpha}\right) \frac{\delta_{0}+\delta_{1}}{\alpha}\right], \\
& R^{\sin \phi_{s}} \approx \frac{2 \alpha}{2 \alpha+\Delta_{2 F}}\left[1+\frac{\Delta_{2 F}}{2 \alpha+\Delta_{2 F}}\left(1-\frac{q_{T}^{2}}{2\left(2 \alpha+\Delta_{2 F}\right)}-\frac{q_{T}^{2}}{4 \alpha}\right) \frac{\delta_{2}}{\alpha}\right], \\
& R^{\sin \left(2 \phi-\phi_{s}\right)} \approx \frac{2 \alpha}{2 \alpha+\Delta_{2 F}}\left[1+\frac{\Delta_{2 F}}{2 \alpha+\Delta_{2 F}}\left(1-\frac{q_{T}^{2}}{2\left(2 \alpha+\Delta_{2 F}\right)}-\frac{q_{T}^{2}}{4 \alpha}\right) \frac{\delta_{3}+\delta_{0}}{\alpha}\right], \\
& R^{\sin \left(2 \phi+\phi_{s}\right)} \approx\left(\frac{2 \alpha}{2 \alpha+\Delta_{2 F}}\right)^{3}\left[1+\frac{\Delta_{2 F}}{2 \alpha+\Delta_{2 F}}\left(2-\frac{q_{T}^{2}}{2\left(2 \alpha+\Delta_{2 F}\right)}-\frac{q_{T}^{2}}{4 \alpha}\right) \frac{\delta_{4}+\delta_{0}}{\alpha}\right] .
\end{aligned}
$$

It is obvious that, only when all the TMD distribution or correlation functions are exactly the same, all the azimuthal asymmetries are suppressed and independent on the transverse momentum. For the general cases, the azimuthal asymmetries depend on both the transverse momentum and the width of the transverse momentum distribution. It turns out that the azimuthal asymmetries of the lepton pair in the nucleon-nucleus process compared with the nucleon-nucleon case can be either suppressed or enhanced. Hence the nuclear modification of the azimuthal asymmetry and its nontrivial transverse momentum dependence provides a very sensitive probe to measure the width of the transverse momentum distribution in the TMD quark distribution functions. For detailed numerical results, we refer the reader to the Ref.. ${ }^{10}$

\section{Acknowledgments}

This work is supported partially by the Major State Basic Research Development Program in China (Grant No. 2014CB845406), the National Natural Science Foundation of China under Grant No. 11475104 and the CCNU-QLPL Innovation Fund (Grant NO. QLPL2014P01).

\section{References}

1. M. Gyulassy and X.-N. Wang, Nucl. Phys. B 420, 583 (1994).

2. R. Baier, Y. L. Dokshitzer, A. H. Mueller, S. Peigne and D. Schiff, Nucl. Phys. B 484, 265 (1997).

3. B. G. Zakharov, JETP Lett. 63, 952 (1996).

4. M. Gyulassy, P. Levai and I. Vitev, Phys. Rev. Lett. 85, 5535 (2000).

5. U. A. Wiedemann, Nucl. Phys. B 588, 303 (2000).

6. X. F. Guo and X.-N. Wang, Phys. Rev. Lett. 85, 3591 (2000).

7. J. H. Gao, Z. T. Liang, X. -N. Wang, Phys. Rev. C81, 065211 (2010).

8. Y. -K. Song, J. -H. Gao, Z. -T. Liang, X. -N. Wang, Phys. Rev. D83, 054010 (2011).

9. J. -H. Gao, A. Schafer and J. Zhou, Phys. Rev. D 85, 074027 (2012). 
10. L. Chen, J. h. Gao and Z. t. Liang, Phys. Rev. C 89, 035204 (2014)

11. J. C. Collins and D. E. Soper, Nucl. Phys. B 193, 381 (1981) [Erratum-ibid. B 213, 545 (1983)] [Nucl. Phys. B 213, 545 (1983)].

12. X. -d. Ji, J. -P. Ma and F. Yuan, Phys. Lett. B 597, 299 (2004)

13. D. Boer, Phys. Rev. D 60, 014012 (1999)

14. S. Arnold, A. Metz and M. Schlegel, Phys. Rev. D 79, 034005 (2009)

15. Z. Lu, B. -Q. Ma and J. Zhu, Phys. Rev. D 84, 074036 (2011)

16. Z. T. Liang, X. N. Wang and J. Zhou, Phys. Rev. D 77, 125010 (2008) 